\title{
Structure of the Kaapvaal craton from surface waves
}

\author{
Marion Freybourger ${ }^{1,2}$, James B. Gaherty ${ }^{3}$, Thomas H. Jordan ${ }^{1,4}$ and the \\ Kaapvaal Seismic Group ${ }^{5}$
}

\begin{abstract}
Phase delays of Rayleigh and Love waves from teleseismic earthquakes recorded during the Southern Africa Seismic Experiment have been inverted for upper-mantle seismic velocities along the propagation paths and beneath the station array. Successful models of subarray structure displayed significant radial anisotropy in the uppermost mantle but no shear-wave low-velocity zone, supporting the hypothesis that the lithosphere beneath the Kaapvaal craton is thick. The azimuthal variations of Rayleigh-wave slowness are not consistent with simple models based on $S K S$ splitting observations and anisotropy measurements of Kaapvaal mantle xenoliths. This discrepancy may imply some amount of small-scale heterogeneity in anisotropy.
\end{abstract}

\section{Introduction}

Southern Africa is a superb natural laboratory for investigating continental structure, comprising a diversity of geological terrains that include the ancient Kaapvaal and Zimbabwe cratons [de Wit et al., 1992]. Kimberlitic mantle xenoliths reveal petrological signatures thought to reflect the chemical stratification and stabilization of a thickened continental tectosphere [e.g., Jordan, 1978]. Recent tomographic studies on global [e.g., Ekström, 1997] and regional scales [e.g., Gaherty and Jordan, 1995; Simons et al., 1999] are consistent with this theory of continental deep structure; they show that the oldest parts of the continents are underlain by a thick (250-350 km) layer of anomalously high seismic velocities and lack the prominent low-velocity zone (LVZ) characteristic of oceanic regions and tectonically active parts of the continent. In a recent study of southern Africa, Zhao et al. [1999] found a one-dimensional (1-D) model with compressional-wave velocities $\sim 3 \%$ higher than global averages down to $300 \mathrm{~km}$. However, earlier dispersion and waveform studies yielded shear-wave models that display a high-velocity lid overlying an oceanic-type LVZ, separated by a Gutenberg discontinuity at $\sim 100$ to $160 \mathrm{~km}$ depth [Bloch et al., 1969; Cichowicz and Green, 1992; Qiu et al., 1996; Priestley, 1999]. As Qiu et al. [1996] (hereon Q96) have noted, the low shear velocities in these latter models are inconsistent with the high velocities inferred for mantle

\footnotetext{
${ }^{1}$ Earth, Atmospheric and Planetary Sciences, Massachusetts Institute of Technology, Cambridge.

${ }^{2}$ now at Observatoire Midi Pyrénées, UMR 5562, Toulouse, France.

${ }^{3}$ School of Earth and Atmospheric Sciences, Georgia Institute of Technology, Atlanta.

${ }^{4}$ now at Department of Earth Sciences, University of Southern California, Los Angeles.

${ }^{5}$ http://www.ciw.edu/kaapvaal
}

Copyright 2001 by the American Geophysical Union.

Paper number 2000 GL012436

0094-8276/01/2000GL012436\$05.00 compositions derived from kimberlitic xenoliths. Perhaps this comparison is misleading or the LVZ beneath southern Africa is a relatively recent feature, postdating the Cretaceous eruption of the kimberlites (e.g., related to the Neogene uplift of the southern Africa plateau).

Alternatively, these isotropic models may not fully account for the structural complexities of southern Africa. Seismic anisotropy has been observed in the region, both on a continental scale [Hadiouche et al., 1989] and locally [Vinnik et al., 1995], and it has long been recognized that anisotropy can bias isotropic models based on multi-component data [e.g., Anderson and Dziewonski, 1982]. In this context, we have used surface-wave data from the Southern Africa Seismic Experiment (SASE) [James et al., 2001] to constrain the anisotropic structure of the southern African upper mantle.

\section{Data Processing and Inversion}

To maximize the resolution of structure in the vicinity of the seismic stations, we selected 10 well-recorded earthquakes at northeastern and southwestern back-azimuths, which had propagation paths sub-parallel to the strike of the station array $\left(\sim \mathrm{N} 35^{\circ} \mathrm{E}\right)$. The epicentral distances ranged from $15^{\circ}$ to $70^{\circ}$ (Fig. 1). The phase delays of the fundamental-mode Rayleigh and Love waves were measured relative to the Preliminary Reference Earth Model (PREM [Dziewonski and Anderson, 1981]). Frequency-dependent travel times were obtained by the GSDF method of Gee and Jordan [1992], in which observed seismograms were cross-correlated with isolation filters calculated by mode summation for target wave groups. This procedure allowed consistent measurement of the phase delays, even in cases where the target wave groups comprised significant higher-mode energy. The GSDF method was adapted to the analysis of array data through the use of an optimized time window found by maximizing the stacks of windowed broadband crosscorrelograms for a range of possible slownesses and group delays. More than 3000 delays were obtained at a total of 65 station sites at 5-millihertz $(\mathrm{mHz})$ intervals from 10 to $45 \mathrm{mHz}$.

The data were jointly inverted for a set of 10 spherically symmetric, path-averaged models (representing the structure from each epicenter to the center of the array) and for a single spherically symmetric model of the structure beneath the array (the subarray model). This procedure minimized the erroneous mapping of path-dependent heterogeneities into the subarray model as well as the errors in the location of the hypocenters. Each model was represented as a radially anisotropic perturbation to PREM, involving two $P$ and $S$ velocities ( $v_{P V}$ and $v_{S V}$ for vertically polarized waves; $v_{P H}$ and $v_{S H}$ for horizontally polarized waves), the mass density $\rho$, and the anisotropic parameter $\eta$. We employed a linearized Gaussian-Bayesian inversion scheme that allowed various constraints to be placed on the models, which included prior estimates of the crustal structure (de- 


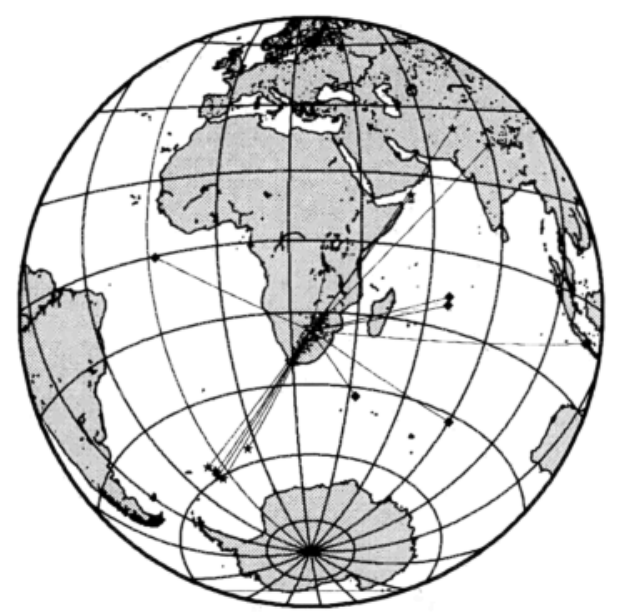

Figure 1. Lambert projection map showing the SASE stations $(\triangle)$, epicenters of earthquakes used in the inversion for the subarray model $(\star)$, and epicenters used to investigate the azimuthal variations $(\diamond)$.

rived from CRUST5.1 [Mooney et al., 1998]) and restricted the models to be PREM-like below $220 \mathrm{~km}$. Because the $P$ velocities in the mantle are poorly determined by the fundamental-mode data, the $v_{P} / v_{S}$ ratio in the uppermost mantle was fixed at 1.77 . The linearized inversion provided a good overall fit to the data set; the phase-delay residuals showed some systematic variations across the array, primarily owing to lateral heterogeneity associated with the structural transitions from the thick, high-velocity cratons to the lower velocity mobile belts, but these deviations from a 1D approximation to subarray structure were generally less than $2 \mathrm{~s}$. The 1-D subarray model was refined by iterating the fit to the data using an exact calculation of the phase velocities.

\section{Radially Anisotropic Models}

Some examples of successful models and their fits to the slowness residuals are illustrated in Fig. 2. Our preferred structure (KA1) has a crustal thickness of $40 \mathrm{~km}$ and average crustal velocities of $v_{S}=3.75 \mathrm{~km} / \mathrm{s}$ and $v_{P}=6.56 \mathrm{~km} / \mathrm{s}$, in agreement with previous studies [e.g., Zhao et al., 1999 and references therein] as well as SASE receiver-function estimates [Nguri et al., 2001]. From the Moho discontinuity (M) to $220 \mathrm{~km}, \mathrm{KA1}$ displays mean velocities larger than PREM (Fig. 3). In the range from a Hales discontinuity (H) at $100 \mathrm{~km}$ depth to $220 \mathrm{~km}$, KA1 velocities are higher than the southern African models that display LVZs. KA1 is anisotropic from $\mathrm{M}$ to $\mathrm{H}$; the magnitude of the anisotropy decreases linearly from $5.6 \%$ to $2.4 \%$ over this depth range. An overall measure of the anisotropy is the horizontal splitting strength, defined to be the depth integral of the slowness difference, $v_{S V}^{-1}-v_{S H}^{-1}$; for KA1, this value is $0.54 \mathrm{~s}$, corresponding to an average $S$-wave anisotropy of 4.1\%. The anisotropy terminates at $\mathrm{H}$, where the average values of $v_{S}$ and $v_{P}$ increase by $2.3 \%$ to values of $4.72 \mathrm{~km} / \mathrm{s}$ and $8.36 \mathrm{~km} / \mathrm{s}$, respectively. While $\mathrm{H}$ is preferred by the data, its depth and sharpness are not well resolved. Below $\mathrm{H}, v_{S}$ decreases slightly to $4.61 \mathrm{~km} / \mathrm{s}$ at $220 \mathrm{~km}$, and $v_{P}$ increases to $8.53 \mathrm{~km} / \mathrm{s}$, although a data-sensitivity analysis shows that neither of these gradients is significantly differ- ent from zero. The seismic velocities are continuous across $220 \mathrm{~km}$ depth.

We have used the data sensitivity kernels in conjunction with a series of forward-modeling experiments to examine the nonuniqueness of the subarray model. Examples of perturbations allowed by the data include an extension of the anisotropy to $120 \mathrm{~km}$ (model KA2 in Fig. 2) and a 0.1-km/s decrease in the upper-crust $P$-wave velocity (KA3). The marginal uncertainties in the average $S$-wave velocities in the two upper-mantle layers above $220 \mathrm{~km}$ are about $1 \%$, and the average $S$-wave anisotropy in the layer between $\mathrm{M}$ and $H$ is constrained to be $4 \pm 1 \%$.

We could not find any isotropic model that both fit the data and was geophysically reasonable. Isotropic models
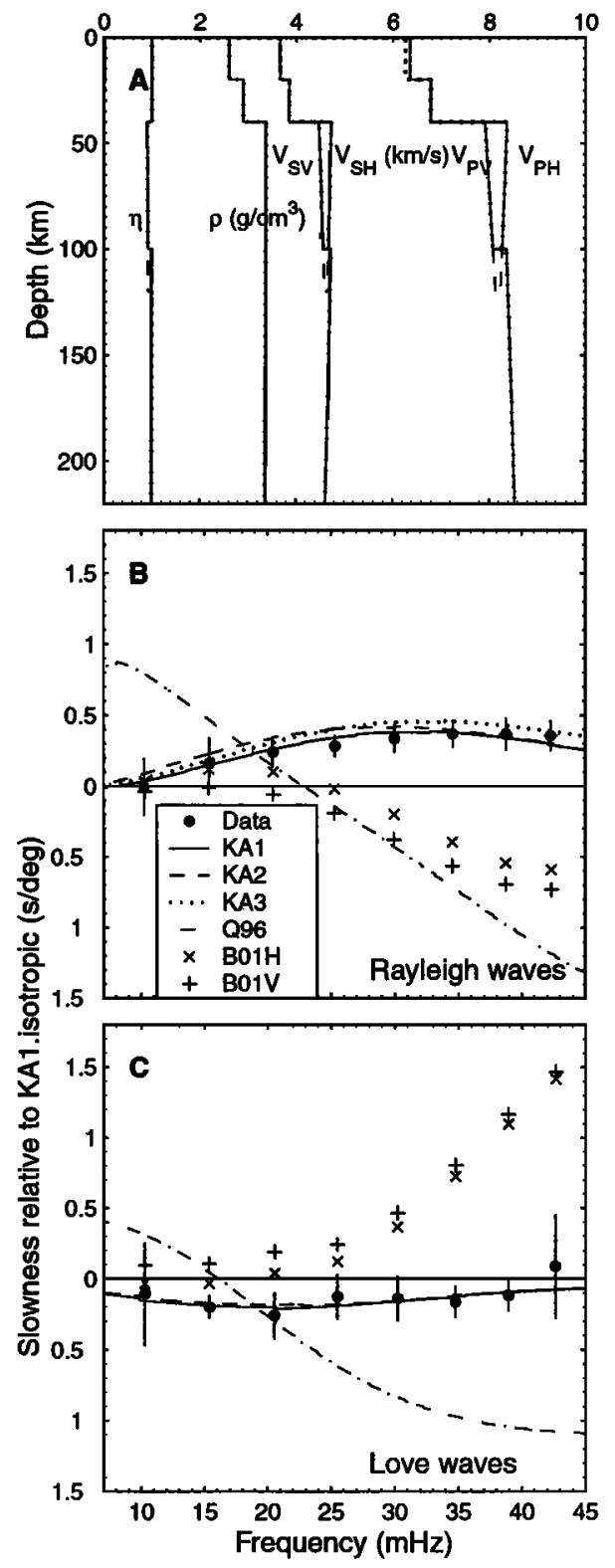

Figure 2. (A) Models KA1, KA2 and KA3, which exemplify radially anisotropic structures that adequately fit the surface-wave dispersion data. (B) Rayleigh-wave dispersion data for the SASE array (solid circles with one-sigma error bars), plotted as slowness residuals relative to KA1.isotropic (mean of model KA1), showing the fit of various models discussed in the text. (C) Same as (B), but for Love waves. See text for further details. 
were constructed that gave adequate fits to the data, but unreasonably low upper-crustal $P$ velocities $(<4 \mathrm{~km} / \mathrm{s}$ in the upper $20 \mathrm{~km}$ ) were required to increase the slowness of the Rayleigh waves relative to the Love waves. An example of an unsuccessful isotropic structure is the model of Q96, which is slow relative to the data at low frequencies and fast at high frequencies (Figs. 2B \& 2C).

\section{Modeling of Azimuthal Anisotropy}

Model KA1 is transversely isotropic, so it does not represent the azimuthal anisotropy observed in the splitting of shear body waves. Measurements of $S K S$ splitting at SASE stations show a coherent pattern of azimuthal anisotropy with an average delay time (vertical splitting strength) of $0.62 \pm 0.02 \mathrm{~s}$ and an alignment of the fast splitting direction subparallel to the axis of the SASE array [Silver et al., 2001, hereon S01]. The vertical and horizontal splitting strengths for the Kaapvaal upper mantle, which measure different aspects of the anisotropy, are therefore comparable. However, both are about $40 \%$ lower than the global mean $(\sim 1 \mathrm{~s}$ for $S K S$ splitting times [Silver, 1996] and the horizontal splitting strength calculated for PREM). This is also in agreement with the laboratory work of Ben-Ismail et al. [2001] (hereon B01), who found that Kaapvaal mantle xenoliths are overall less anisotropic than peridotites measured from other localities.

To investigate the effect of azimuthal anisotropy on the surface waves, we used first-order perturbation theory [Montagner and Nataf, 1986] to calculate the Rayleigh and Love phase velocities for transversely anisotropic structures that satisfied the average $S K S$ splitting observations and had the same isotropic average as KA1. We used B01's average of the elastic tensors from 48 Kaapvaal xenoliths to construct two structures, one with a horizontal foliation plane (B01-H) and one with a vertical foliation plane (B01-V). We scaled the anisotropic-layer thickness by requiring a vertical splitting strength of $0.62 \mathrm{~s}$, resulting in 110 and $168 \mathrm{~km}$ respectively. For surface waves propagating along the axis of the array (i.e., near the fast axis of the $S K S$ splitting), the B01 anisotropy predicted negative perturbations to the Rayleigh-

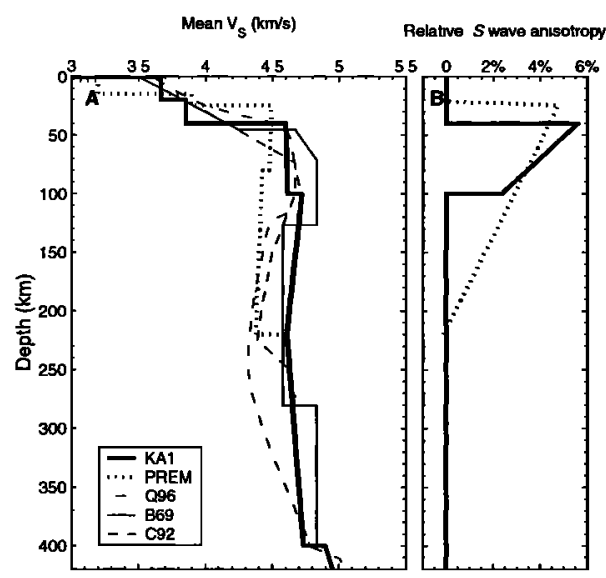

Figure 3. Comparison of KA1 with PREM and the isotropic upper-mantle models of Bloch et al. [1969] (B69, their SASD3 model), Cichowicz and Green [1992] (C92), and Q96. (A) Mean shear velocity $\bar{v}_{S}=\left(v_{S H}+v_{S V}\right) / 2$. (B) Relative shear-wave anisotropy, $\left(v_{S H}-v_{S V}\right) / \bar{v}_{S}$.

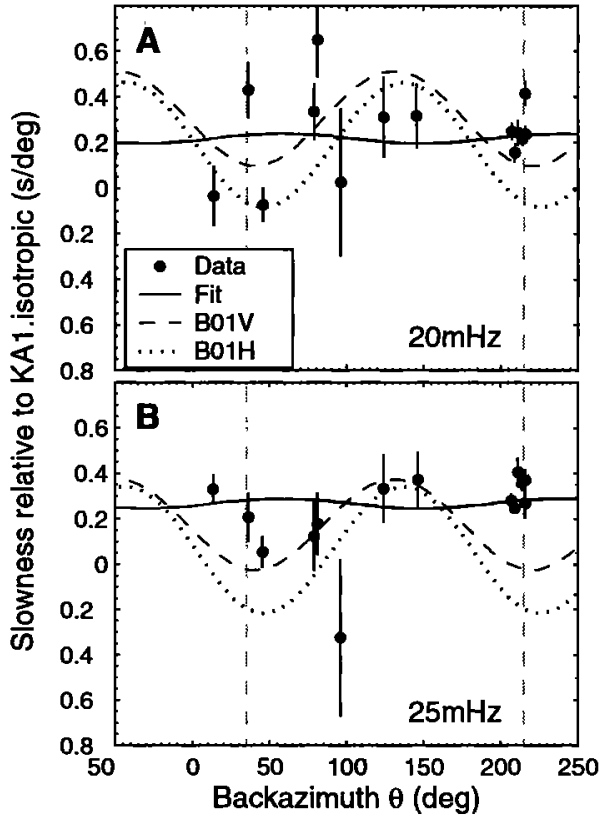

Figure 4. Azimuthal variation of slowness data for the SASE array (solid circles with one-sigma error bars), plotted as residuals relative to KA1.isotropic, for Rayleigh waves at frequencies of $20 \mathrm{mHz}$ (A) and $25 \mathrm{mHz}$ (B). Solid lines show the least-squares fit of the function $A \cos \left(2\left(\theta-45^{\circ}\right)\right)+B$ to the data, which gave $A=-0.02 \pm 0.01 \mathrm{~s} /{ }^{\circ}$. Other sinusoids are from the models described in Fig. 2, B01-V (dashed lines) and B01-H (dotted lines); these models predict azimuthal variations significantly larger than observed at these frequencies. The vertical dashed lines mark the approximate strike of the SASE array.

wave slowness and positive perturbations to the Love-wave slowness, opposite in sign to the data residuals (Figs. 2B \& 2C). This reversal in sign of the Rayleigh and Love slowness perturbations for propagation directions along the fast axis of the anisotropy was pointed out by Maupin [1985]. A homogeneous model of Kaapvaal anisotropy based on xenolith measurements was thus unable to satisfy both the surfacewave dispersion and $S K S$ splitting measurements.

The transversely anisotropic models also predicted azimuthal variations in Rayleigh-wave slowness. To look for these variations, we measured the Rayleigh-wave slownesses for an additional six earthquakes with back-azimuths at high angles to the array axis (Fig. 1). The observations at 20 and $25 \mathrm{mHz}$, where the signal-to-noise ratios were highest, are compared with the B01 model values in Fig. 4. The additional data showed substantial scatter, owing in part to the difficulty in measuring apparent slowness at the smaller array apertures for off-axis arrivals and also to lateral refraction and multipathing caused by aspherical heterogeneities. However, they did not display the $\cos (2 \theta)$ variation implied by the B01 models, which predict higher values of slowness perpendicular to the array axis; a least-squares fit to the data in Fig. 4 yielded an amplitude of $-0.02 \pm 0.01 \mathrm{~s} /{ }^{\circ}$, compared to $+0.20 \mathrm{~s} /{ }^{\circ}$ for $\mathrm{B} 01-\mathrm{V}$ and $+0.28 \mathrm{~s} /{ }^{\circ}$ for $\mathrm{B} 01-\mathrm{H}$. Other published tensors [e.g., Peselnick and Nicolas, 1978] typically predicted even larger variations ( 0.3-0.4 $\left.\mathrm{s} /{ }^{\circ}\right)$. The lack of any resolvable azimuthal variation in our data is also inconsistent with the magnitude of the azimuthal anisotropy in southern Africa mapped on a continental scale by Hadiouche et al. [1989]. 


\section{Discussion and Conclusions}

Several important conclusions can be drawn from this preliminary analysis of surface-wave dispersion across the SASE array. The mantle beneath the Kaapvaal craton is characterized by high seismic velocities; there is no evidence for a substantial LVZ in the upper $200 \mathrm{~km}$. The data require radial anisotropy in the uppermost mantle; the magnitude of the $S$-wave anisotropy at the top of the mantle $(\sim 4 \%)$ is comparable to values found elsewhere, but the thickness of the anisotropic layer $(<100 \mathrm{~km})$ appears to be less than in other regions, such as Australia [Gaherty and Jordan, 1995]. No significant azimuthal anisotropy was observed in lowfrequency (20-25 $\mathrm{mHz}$ ) Rayleigh waves, and we were unable to satisfy the surface-wave data with the type of homogeneous, transversely anisotropic structure used to explain the $S K S$ splitting observations. If $S K S$ splitting is related to lithospheric fabric [S01] as opposed to asthenospheric fabric [Vinnik et al., 1995], one has to invoke some kind of heterogeneity to explain the lack of resolvable azimuthal variations in the Rayleigh wave data. Both lateral and vertical heterogeneity in the local orientation of the elastic tensor can act to reduce the azimuthal anisotropy of the low-frequency surface waves while preserving (or even increasing) the amount of the polarization anisotropy [Jordan and Gaherty, 1995]. The relatively constant $S K S$ splitting orientations observed by S01 limit the amount of lateral heterogeneity, however. Some degree of vertical heterogeneity may be thus required to reconcile the two types of data. Under a single-scattering approximation, the splitting amplitude of $S K S$ waves is essentially insensitive to vertical variations in the orientation of the local fast axis $\phi(z)$ [e.g., Saltzer et al., 2000]. The amplitude of surface-wave azimuthal anisotropy will depend on a depth integral of $\cos (2 \phi(z))$ and will therefore tend to average to zero if the variation in $\phi(z)$ is large. It remains to be seen whether consistent models based on stochastic representations of heterogeneities and scattering theory can be found.

Acknowledgments. We thank the Kaapvaal Seismic Group for making this project successful; in particular Rod Green, Jane Goré, Josh Harvey, Randy Kuehnel, Andrew Menzies, Teresia Nguuri, Jock Robie, Rebecca Saltzer, Sue Webb, Kalle Westerlund, and many others who installed and maintained the instruments and collected the data during the two-year experiment. We also thank $\mathrm{Li}$ Zhao for numerous fruitful discussions. This research was sponsored by the NSF under grant EAR-9526702.

\section{References}

Anderson, D. L., and A. M. Dziewonski, Upper mantle anisotropy; evidence from free oscillations, Geophys. J. R. astron. Soc., 69, 383-404, 1982.

Ben-Ismaill, W., G. Barruol, and D. Mainprice, The Kaapvaal craton seismic anisotropy: a petrophysical analysis of upper mantle kimberlite nodules, Geophys. Res. Lett., 2001, this issue.

Bloch, S., A. L. Hales, and M. Landisma, Velocities in the crust and upper mantle of southern Africa from multimode surface wave dispersion, $B S S A, 59,1599-1629,1969$.

Cichowicz, A., and R. W. E. Green, Tomographic structure of the upper-mantle structure of the South African continent, using wave-form inversion, Phys. Earth Planet. Int., 72, 276-285, 1992.

de Wit, M. J., C. Roering, R. J. Hart, R. A. Armstrong, C. E. J. de Ronde, R. W. E. Green, M. Tredoux, E. Peberdy, and R. A. Hart, Formation of an archean continent, Nature, 357, 553$562,1992$.

Dziewonski, A. M., and D. L. Anderson, Preliminary Reference Earth Model, Phys. Earth Planet. Int., 25, 297-356, 1981.
Ekström, G., J. Tromp, and E. W. F. Larson, Measurements and global models of surface wave propagation, J. Geophys. Res., 102, 8137-8157, 1997.

Gaherty, J. B., and T. H. Jordan, Lehmann discontinuity as the base of an anisotropic layer beneath continents, Science, 268, 1468-1471, 1995.

Gee, L. S., and T. H. Jordan, Generalized seismological data functionals, Geophys. J. Int., 111, 363-390, 1992.

Hadiouche, O., N. Jobert, and J.-P. Montagner, Anisotropy of the African continent inferred from surface waves, Phys. Earth Planet. Int., 58, 61-81, 1989.

James, D. E., M. J. Fouch, J. C. VanDecar, S. van der Lee, and the Kaapvaal Seismic Group, Tectospheric structure beneath southern Africa, Geophys. Res. Lett., 2001, this issue.

Jordan, T. H., Composition and development of the continental tectosphere, Nature, 274, 544-548, 1978.

Jordan, T. H., and J. B. Gaherty, Stochastic modeling of smallscale, anisotropic structures in the continental upper mantle, in Proc. 17th Ann. Seismic Res. Symp., edited by J. F. Lewkowicz, J. M. McPhetre and D. T. Reide, pp. 433-451, Phillips Labs, Hanscom Air Force Base, 1995.

Maupin, V., Partial derivatives of surface wave phase velocities for flat anisotropic models, Geophys. J. R. astron. Soc., 83, 379-398, 1985.

Montagner, J.-P., and H.-C. Nataf, A simple method for inverting the azimuthal anisotropy of surface waves, J. Geophys. Res., 91, 511-520, 1986.

Mooney, W. D., G. Laske, and T. G. Masters, Crust 5.1: A global crustal model at $5^{\circ} \times 5^{\circ}, J$. Geophys. Res., 103, 727-747, 1998.

Nguuri, T. K., J. Goré, D. E. James, C. Wright, T. G. Zengeni, O. Gwavava, S. J. Webb, J. A. Snoke, and the Kaapvaal Seismic Group, Crustal structure beneath southern Africa and its implications for the formation and evolution of the Kaapvaal and Zimbabwe cratons, Geophys. Res. Lett., 2001, this issue.

Peselnick, L., and A. Nicolas, Seismic anisotropy in an ophiolite peridotite: application to oceanic upper mantle, J. Geophys. Res., 83, 1227-1235, 1978.

Priestley, K., Velocity structure of the continental upper mantle: evidemce from southern Africa, Lithos, 48, 45-56, 1999.

Qiu, X., K. Priestley, and D. McKenzie, Average lithospheric structure of southern Africa, Geophys. J. Int., 127, 563-587, 1996.

Saltzer, R. L., J. B. Gaherty, and T. H. Jordan, How are vertical shear wave splitting measurements affected by variations in the orientation of azimuthal anisotropy with depth?, Geophys. J. Int., 141, 374-390, 2000.

Silver, P. G., Seismic anisotropy beneath the continents: probing the depths of geology, Ann. Rev. Earth Planet. Sci., 24, 385432, 1996.

Silver, P. G., S. S. Gao, K. H. Liu, and the Kaapvaal Seismic Group, Mantle deformation beneath southern Africa, Geophys. Res. Lett., 2001, this issue.

Simons, F. J., A. Zielhuis, and R. D. van der Hilst, The deep structure of the Australian continent from surface-wave tomography, Lithos, 48, 17-43, 1999.

Vinnik, L. P., R. W. E. Green, and L. O. Nicolaysen, Recent deformations of the deep continental root beneath southern Africa, Nature, 375, 50-52, 1995.

Zhao, M., C. A. Langston, A. A. Nyblade, and T. J. Owens, Upper mantle velocity structure beneath southern Africa from modeling regional seismic data, Geophys. J. Int., 129, 412420,1999 .

M. Freybourger, UMR 5562, Observatoire Midi Pyrénées, 14 avenue Édouard Belin, F-31400 Toulouse, France

(Marion.Freybourger@cnes.fr).

J. Gaherty, School of Earth and Atmospheric Sciences, Georgia Institute of Technology, Atlanta, GA 30332

(gaherty@eas.gatech.edu).

T. Jordan, Department of Earth Sciences, USC, Los Angeles, CA 90089 (tjordan@usc.edu).

(Received October 3, 2000; revised March 14, 2001; accepted March 21, 2001.) 